\title{
PARTIAL CLOSURE OF CRACKS AT THE INTERFACE BETWEEN A LAYER AND A HALF SPACE
}

\author{
MARIA COMNINOU \\ Department of Civil Engineering, University of Michigan, Ann Arbor, MI 48109, U.S.A. \\ and \\ JOHN DUNDURS \\ Department of Civil Engineering, Northwestern University, Evanston, IL 60201, U.S.A.
}

\begin{abstract}
Ahstract-The paper treats the crack at the interface between a layer and a substrate. Using a formulation based on dislocations, the problem is reduced to a pair of singular integral equations which can be solved numerically. It is found that a large contact zone exists whenever the interface transmits shear. The dependence of the contact zone size on the load parameters is determined for several combinations of materials. Also the shapes of the open part of the crack are shown for illustration.
\end{abstract}

\section{INTRODUCTION}

THE PARTIAL closure of an interface crack between two elastic unbounded solids has been studied in recent publications [1-5] with or without friction. It was found that if the applied loads cause predominantly shear tractions to be transmitted by the interface, then a large part of the crack, often of the order of a third of the crack length, remains closed. Significant closure has also been observed experimentally by Janach and Guex[6], and Liechti and Knauss [7]. This phenomenon can be directly attributed to the presence of shear and it is not related to the problems of oscillatory singularities and the ensuing material interpenetration at the crack tips. The latter can be avoided by requiring that the crack tips be closed $[1,8]$ in the context of linear elasticity. The extent of the associated contact zones in the vicinity of the tips is extremely small in comparison to the crack length (of the order of $10^{-6}$ ) for purely tensile loading. It should also be noted that oscillatory singularities do not occur at the tips of cracks at the interface between nonlinear materials, as shown by Knowles and Sternberg [9].

As mentioned before, the partial closure due to shear is not related to the fact that small contact zones must exist to prevent oscillatory singularities, and it occurs only on one side of the crack depending on the direction of shear and the relative rigidity of the two solids. On the other side, the crack tip must be also closed to avoid material interpenetration in the context of linear elasticity, but the extent of this contact zone is essentially infinitesimal as in the case of pure tension, and can probably be disregarded.

In this paper we investigate the effect of a finite boundary on the partial closure of an interface crack. For simplicity, we consider an elastic layer bonded to a half space of different material properties. A crack of finite length is situated at the interface. The layer is loaded by uniform shear and compression (uniform tension is also considered for comparison purposes) and the effect of these loads on the extent of the contact zones that develop is studied. The effect of the thickness of the layer relative to the crack length is also considered.

\section{THE DISLOCATION SOLUTION}

We intend to formulate the problem using distributed dislocations to model the crack as in [1-5]. We, therefore, need the solution for a discrete dislocation with Burgers vector $\left(b_{x}, b_{y}\right)$ situated at the origin of the coordinate system which is placed at the interface between the layer and the substrate, as shown in Fig. 1. In particular we need the tractions at the interface due to the dislocation. The interface tractions for a climb dislocation (Burgers vector $\left.\left(0, b_{y}\right)\right)$ can be obtained from Weeks et al.[10] after solving a linear system of six algebraic equations for six unknowns. The solution for a glide dislocation (Burgers vector $\left(b_{x}, 0\right)$ ) is constructed following the analysis of [10]. The idea is basically to start with the known solution for a dislocation near the interface between two bonded half planes, compute the tractions transmitted by the joined half planes at the position where the free surface of the layer is going to be and 


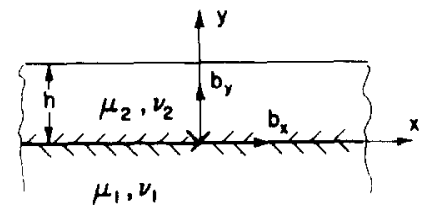

Fig. 1. Dislocation at the interface of a layer and a half plane.

cancel these tractions by a Fourier transform solution. Care must be taken in the computation of the interface tractions when the dislocation itself is at the interface, so that the singular behavior of the interface tractions is retained correctly[11]. Only the results are quoted here.

The interface tractions due to a climb dislocation at the origin are

$$
\begin{gathered}
\sigma_{x y}(x, 0)=\frac{\mu_{1}(1+\alpha) b_{y}}{\pi\left(\kappa_{1}+1\right)\left(1-\beta^{2}\right)}\left[2 \pi \beta \delta(x)-\frac{1-\alpha}{h} \int_{0}^{\infty} \frac{N_{12}}{D} \mathrm{e}^{-t} \cos \frac{x t}{h} \mathrm{~d} t\right] \\
\sigma_{y y}(x, 0)=\frac{\mu_{1}(1+\alpha) b_{y}}{\pi\left(\kappa_{1}+1\right)\left(1-\beta^{2}\right)}\left[\frac{2}{x}-\frac{1-\alpha}{h} \int_{0}^{\infty} \frac{N_{11}}{D} \mathrm{e}^{-t} \sin \frac{x t}{h} \mathrm{~d} t\right]
\end{gathered}
$$

The interface tractions due to a glide dislocation at the origin are

$$
\begin{aligned}
& \sigma_{x y}(x, 0)=\frac{\mu_{1}(1+\alpha) b_{x}}{\pi\left(\kappa_{1}+1\right)\left(1-\beta^{2}\right)}\left[\frac{2}{x}-\frac{1-\alpha}{h} \int_{0}^{\infty} \frac{N_{22}}{D} \mathrm{e}^{-t} \sin \frac{x t}{h} \mathrm{~d} t\right] \\
& \sigma_{y y}(x, 0)=\frac{\mu_{1}(1+\alpha) b_{x}}{\pi\left(\kappa_{1}+1\right)\left(1-\beta^{2}\right)}\left[-2 \pi \beta \delta(x)+\frac{1-\alpha}{h} \int_{0}^{\infty} \frac{N_{22}}{D} \mathrm{e}^{-t} \cos \frac{x t}{h} \mathrm{~d} t\right] .
\end{aligned}
$$

In eqns (1)-(4) $h$ is the thickness of the layer, $\mu$ is the shear modulus, $\kappa=3-4 \nu$, where $\nu$ is Poisson's ratio and the constants $\alpha$ and $\beta$ are defined[12] as

$$
\alpha=\frac{\mu_{2}\left(\kappa_{1}+1\right)-\mu_{1}\left(\kappa_{2}+1\right)}{\mu_{2}\left(\kappa_{1}+1\right)+\mu_{1}\left(\kappa_{2}+1\right)}, \quad \beta=\frac{\mu_{2}\left(\kappa_{1}-1\right)-\mu_{1}\left(\kappa_{2}-1\right)}{\mu_{2}\left(\kappa_{1}+1\right)+\mu_{1}\left(\kappa_{2}+1\right)} .
$$

Finally, $D, N_{12}, N_{11}, N_{22}$ are the following functions of the argument $t$

$$
\begin{aligned}
D & =\left(1-\beta^{2}\right) \mathrm{e}^{2 t}+\left(\alpha^{2}-\beta^{2}\right) \mathrm{e}^{-2 t}-4(1+\beta)(\alpha-\beta) t^{2}-2\left(\alpha-\beta^{2}\right) \\
N_{12} & =4\left[\left(1+\beta^{2}\right) t^{2}+\beta\right] \mathrm{e}^{t}-2 \beta(1+\alpha) \mathrm{e}^{-t} \\
N_{11} & =2\left\{2(1+\beta)[(1+\beta) t+1-\beta] t+1+\beta^{2}\right\} \mathrm{e}^{t}-2\left(\alpha+\beta^{2}\right) \mathrm{e}^{-t} \\
N_{22} & =2\left\{2(1+\beta)[(1+\beta) t-(1-\beta)] t+1+\beta^{2}\right\} \mathrm{e}^{t}-2\left(\alpha+\beta^{2}\right) \mathrm{e}^{-t} .
\end{aligned}
$$

For identical materials the solution given here coincides with that obtained from [12] as a special case of a dislocation near a phase boundary.

\section{FORMULATION}

Consider a crack of length $2 L$ at the interface between the layer and the substrate, Fig. 2 . The surface of the layer is loaded by given tractions so that the bond, in absence of the crack, transmits the

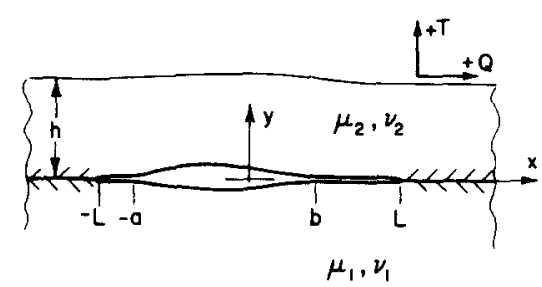

Fig. 2. The interface crack between a layer and a half plane. 
normal and shear tractions $T(x)$ and $Q(x)$. The crack will be partially closed and at least two contact zones will develop in the vicinity of the crack tips, as explained in the Introduction. The possibility of a third, detached contact zone cannot be excluded, but it will not be analyzed here, although the conditions for its occurrence will be discussed later. The two contact zones, then, occupy the interval $(-L,-a)$ and $(b, L)$, while $(-a, b)$ is the interval of the gap defined as

$$
g(x)=u_{y}\left(x, 0^{+}\right)-u_{y}\left(x, 0^{-}\right),-a<x<b .
$$

The tangential shift along the crack is defined as

$$
h(x)=u_{x}\left(x, 0^{+}\right)-u_{x}\left(x, 0^{-}\right), \quad-L<x<L .
$$

The gap can be modelled by a distribution of climb dislocations with density $B_{y}(x)$, and the tangential shift by a distribution of glide dislocations with density $B_{x}(x)$ so that

$$
B_{y}(x)=-\frac{\mathrm{d} g}{\mathrm{~d} x}, B_{x}(x)=-\frac{\mathrm{d} h}{\mathrm{~d} x}
$$

Note that $B_{y}(x)$ is defined over $(-a, b)$, while $B_{x}(x)$ is defined over $(-L, L)$ and they are zero everywhere else. The interface tractions due to these distributions are obtained by integration from eqns (1)-(4). The total tractions are then

$$
\begin{aligned}
& N(x)=T(x)+C\left\{-\beta B_{x}(x)-\frac{1}{\pi} \int_{-a}^{b} \frac{B_{y}(\xi)}{\xi-x} \mathrm{~d} \xi+\frac{1-\alpha}{2 \pi h}\left[\int_{-a}^{b} B_{y}(\xi) K_{1}(\xi, x) \mathrm{d} \xi+\int_{-L}^{L} B_{x}(\xi) K_{3}(\xi, x) \mathrm{d} \xi\right]\right\} \\
& S(x)=Q(x)+C\left\{\beta B_{y}(x)-\frac{1}{\pi} \int_{-L}^{L} \frac{B_{x}(\xi)}{\xi-x} \mathrm{~d} \xi+\frac{1-\alpha}{2 \pi h}\left[\int_{-L}^{L} B_{x}(\xi) K_{2}(\xi, x) \mathrm{d} \xi-\int_{-a}^{b} B_{y}(\xi) K_{3}(\xi, x) \mathrm{d} \xi\right]\right\}
\end{aligned}
$$

where

$$
\begin{aligned}
& C=\frac{2 \mu_{1}(1+\alpha)}{\left(\kappa_{1}+1\right)\left(1-\beta^{2}\right)}=\frac{2 \mu_{2}(1-\alpha)}{\left(\kappa_{2}+1\right)\left(1-\beta^{2}\right)} \\
& K_{1}(\xi, x)=\int_{0}^{\infty} \frac{N_{11}}{D} \mathrm{e}^{-t} \sin \frac{(\xi-x) t}{h} \mathrm{~d} t \\
& K_{2}(\xi, x)=\int_{0}^{\infty} \frac{N_{22}}{D} \mathrm{e}^{-t} \sin \frac{(\xi-x)}{h} \mathrm{dt} \\
& K_{3}(\xi, x)=\int_{0}^{\infty} \frac{N_{12}}{D} \mathrm{e}^{-t} \cos \frac{(\xi-x)}{h} \mathrm{dt}
\end{aligned}
$$

The boundary conditions are

$$
\begin{aligned}
& N(x)=0-a<x<b \\
& S(x)=0-L<x<L
\end{aligned}
$$

yielding two coupled singular integral equations of the Cauchy type for the unknown densities $B_{x}(x)$ and $B_{y}(x)$. In addition, the following conditions must be imposed to avoid multivalued displacements

$$
\int_{-a}^{b} B_{y}(\xi) \mathrm{d} \xi=0, \quad \int_{-L}^{L} B_{x}(\xi) \mathrm{d} \xi=0
$$

The singular integral equations together with (22) and (23) are sufficient for the determination of $B_{x}(x)$, $B_{y}(x)$ and of the unknown parameters $a$ and $b$. 
In the subsequent numerical computations we consider as example the case of uniform applied tractions so that

$$
T(x)=T=\text { const }, Q(x)=Q=\text { const }
$$

where $T>0$ corresponds to tension and $Q>0$ points to the right on the surface of the layer. Recalling that one contact zone is expected to be infinitesimal and the other large in extent, we fix $\beta Q>0$. This has the effect that the large contact zone always appears at the right tip[1], and the discretization procedure is simplified without loss of generality.

\section{DISCRETIZATION AND NUMERICAL SOLUTION}

First we normalize each of the intervals $(-a, b),(-L, L)$ by the following changes of variables

$$
\xi=L r, \quad x=L s \text { for the interval }(-L, L)
$$

and

$$
\xi=(\delta \hat{r}+\sigma) L, \quad x=(\delta \hat{s}+\sigma) L \text { for the interval }(-a, b)
$$

where

$$
\delta=(b+a) / 2 L, \quad \sigma=(b-a) / 2 L,
$$

and retain the same symbols for the functions in the new variables. Noting that $B_{x}(\xi)$ must be singular at both tips $\pm L$, we let

$$
B_{x}(r)=\frac{Q}{C} \phi(r)\left(1-r^{2}\right)^{-1 / 2},-1<r<1
$$

On the contrary, $B_{y}(x)$ must be bounded at $-a$ and $b[8]$, a condition which is equivalent to requiring that the normal tractions vanish at $-a$ and $b$. However, it has been found [1-4] that, since $-a$ must be very close to $-L$ and the slope of the gap changes rapidly there, it is computationally more advantageous to take $B_{y}(x)$ unbounded at $-a$, as if the gap were open there, and then require that

$$
N(-a)=0 \text { or } B_{y}(-a)=0
$$

so that the intensity of the singularity allowed vanishes at $-a$. Then

$$
B_{y}(\hat{r})=\frac{Q}{C} \psi(\hat{r})(1+\hat{r})^{-1 / 2}(1-\hat{r})^{1 / 2}, \quad-1<\hat{f}<1
$$

In (29) and (31) $\phi(r)$ and $\psi(\hat{r})$ are regular functions and the square root behavior is dictated by the asymptotics [8]. The boundary conditions $(20)$ and $(21)$ become

$$
\begin{aligned}
& -\beta \phi(\hat{s})\left[1-(\delta \hat{s}+\sigma)^{2}\right]^{-1 / 2}-\frac{1}{\pi} \int_{-1}^{1} \frac{\psi(\hat{r})(1-\hat{r})^{1 / 2}}{(\hat{r}-\hat{s})(1+\hat{r})^{1 / 2} \mathrm{~d} \hat{r}} \\
& +\frac{(1-\alpha) L}{2 \pi h}\left\{\delta \int_{-1}^{1} \psi(\hat{r})(1-\hat{r})^{1 / 2}(1+\hat{r})^{-1 / 2} K_{1}(\hat{r}, \hat{s}) \mathrm{d} \hat{r}\right. \\
& \left.+\int_{-1}^{1} \phi(r)\left(1-r^{2}\right)^{-1 / 2} K_{3}(r, \hat{s}) \mathrm{d} r\right\}=-\frac{T}{Q}, \quad-1<\hat{s}<1 \\
& \beta \psi(s)\left[1-\frac{s-\sigma}{\delta}\right]^{1 / 2}\left[1+\frac{s-\sigma}{\delta}\right]^{-1 / 2}\left[H\left(s+\frac{a}{L}\right)-H\left(s-\frac{b}{L}\right)\right] \\
& \quad-\frac{1}{\pi} \int_{-1}^{1} \frac{\phi(r)\left(1-r^{2}\right)^{-1 / 2}}{r-s} \mathrm{~d} r+\frac{(1-\alpha) L}{2 \pi h}\left\{\int_{-1}^{1} \phi(r)\left(1-r^{2}\right)^{-1 / 2} K_{2}(r, s) \mathrm{d} r\right. \\
& \left.-\delta \int_{-1}^{1} \psi(\hat{r})(1-\hat{r})^{1 / 2}(1+\hat{r})^{-1 / 2} K_{3}(\hat{r}, s) \mathrm{d} \hat{r}\right\}=-1, \quad-1<s<1
\end{aligned}
$$

where $H()$ denotes the Heaviside step function. 
To discretize these coupled singular integral equations of Cauchy type, we apply the method of Erdogan and Gupta[13]. Accordingly, the arguments $s$ and $\hat{s}$ are replaced by the collocation points

$$
\begin{aligned}
& s_{k}=\cos \left(\frac{\pi k}{n}\right), \quad k=1, \ldots n-1 \\
& \hat{s}_{k}=\cos \left[\frac{\pi(2 k-1)}{2 n+1}\right], \quad k=1, \ldots n
\end{aligned}
$$

and the integration variables $r$ and $\hat{r}$ are replaced by the integration points

$$
\begin{gathered}
r_{i}=\cos \left[\frac{\pi(2 i-1)}{2 n}\right], \quad i=1, \ldots n \\
\hat{r}_{i}=\cos \left(\frac{2 \pi i}{2 n+1}\right), \quad i=1, \ldots n .
\end{gathered}
$$

The integrals are then replaced by summations according to the corresponding Chebyshev quadratures. A difficulty arises in that the arguments of the functions $\phi$ and $\psi$ in the free terms do not coincide with their arguments under the integration signs. This problem is resolved by using Krenk's interpolation formulas [14] to write

$$
\begin{aligned}
& \phi\left(\hat{s}_{k}\right)=\sum_{i=1}^{n} \Phi_{i}\left(\hat{s}_{k}\right) \phi\left(r_{i}\right) \\
& \psi\left(s_{k}\right)=\sum_{i=1}^{n} \Psi_{i}\left(s_{k}\right) \psi\left(\hat{r}_{i}\right) .
\end{aligned}
$$

Using the discretized form of (22) and (23)

$$
\frac{2 \pi}{2 n+1} \sum_{i=1}^{n}\left(1-\hat{r}_{i}\right) \psi\left(\hat{r}_{i}\right)=0, \quad \frac{\pi}{n} \sum_{i=1}^{n} \phi\left(r_{i}\right)=0
$$

and the formulas given in [4] we obtain the coefficients $\Phi_{i}\left(\hat{s}_{k}\right)$ and $\Psi_{i}\left(s_{k}\right)$

$$
\begin{gathered}
\Phi_{i}\left(\hat{s}_{k}\right)=\frac{2}{n} \sum_{j=1}^{n-1} \cos \left[\frac{2 i-1}{2 n} j \pi\right] T_{j}\left(\delta \hat{s}_{k}+\sigma\right), \quad i=1, \ldots n, \quad k=1, \ldots n \\
\Psi_{i}\left(s_{k}\right)=\frac{4}{2 n+1} \sin \left(\frac{\pi i}{2 n+1}\right) \sum_{j=1}^{n-1} \sin \left[\frac{2 j+1}{2 n+1} i \pi\right] U_{2 j}\left[\sqrt{ }\left(\frac{1}{2}+\frac{s_{k}-\sigma}{2 \delta}\right)\right], \quad i=1, \ldots n, \quad k=1, \ldots n-1
\end{gathered}
$$

where $T_{j}$ and $U_{j}$ are the Chebyshev polynomials of first and second kind. The discretized forms of eqns (32) and (33) are then

$$
\begin{aligned}
& \sum_{i=1}^{n}\left\{\beta\left[1-\left(\delta \hat{s}_{k}+\sigma\right)^{2}\right] \Phi_{i}\left(\hat{s}_{k}\right)-\frac{(1-\alpha) L}{2 h n} K_{3}\left(r_{i}, \hat{s}_{k}\right)\right\} \phi\left(r_{i}\right) \\
& \quad+\sum_{i=1}^{n}\left\{\frac{1}{\hat{r}_{i}-\hat{s}_{k}}-\frac{(1-\alpha) \delta L}{2 h} K_{1}\left(\hat{r}_{i}, \hat{s}_{k}\right)\right\} \frac{2\left(1-\hat{r}_{i}\right)}{2 n+1} \psi\left(\hat{r}_{i}\right)=\frac{T}{Q}, \quad k=1, \ldots n \\
& \sum_{i=1}^{n}\left\{\frac{1}{r_{i}-s_{k}}-\frac{(1-\alpha) L}{2 h} K_{2}\left(r_{i}, s_{k}\right)\right\} \frac{1}{h} \phi\left(r_{i}\right) \\
& \quad+\sum_{k=1}^{n}\left\{-\beta\left[1-\frac{s_{k}-\sigma}{\delta}\right]^{1 / 2}\left[1+\frac{s_{k}+\sigma}{\delta}\right]^{-1 / 2}\left[H\left(s_{k}+\frac{a}{L}\right)-H\left(s_{k}-\frac{b}{L}\right)\right] \Psi_{i}\left(s_{k}\right)\right. \\
& \left.\quad+\frac{(1-\alpha) \delta L\left(1-\hat{r}_{i}\right)}{h(2 n+1)} K_{3}\left(\hat{r}_{i}, s_{k}\right)\right\} \psi\left(\hat{r}_{i}\right)=1, \quad k=1, \ldots n-1 .
\end{aligned}
$$

The discretized form of $(30)$ is

$$
\left.\psi(\hat{r})\right|_{\hat{r}=-1}=0
$$


or, using the interpolation formulas [14],

$$
\frac{2}{2 n+1} \sum_{i=1}^{n} \cot \left[\frac{(2 i-1) \pi}{2(2 n+1)}\right] \sin \left[\frac{n(2 i-1) \pi}{2 n+1}\right] \psi\left(\hat{r}_{n+1-i}\right)=0
$$

Equations (44), (45), (47), (40) and (41) provide a system of $2 n+2$ equations for the $2 n+2$ unknowns $\phi\left(r_{i}\right), \psi\left(\hat{r}_{i}\right), a$ and $b$. The equations are linear in all the unknowns except $a$ and $b$. Moreover, $-a$ must be very close to $-L$, and the precise computation of its value is not expected to affect significantly the other quantities of interest $[1,2]$. To further simplify the numerical procedure we interchange the roles of $b / L$ and $T / Q$. Thus we treat $b / L$ as a known (specified) quantity and derive $T / Q$ from the solution of the system. When this is done, eqns (44), (45), (40) and (41) provide a system of $2 n+1$ linear equations with $2 n+1$ unknowns $\phi\left(r_{i}\right), \psi\left(\hat{r}_{i}\right)$ and $T / Q$ for each value of $a$. The parameter $a$ is then obtained by iteration using (47). For a chosen value of $a$ the system of $2 n+1$ equations is solved and the known values of $\psi\left(\hat{r}_{i}\right)$ are substituted into (47) which is not generally satisfied. A new guess is made for $a$ and the process repeated until (47) is satisfied. In practice it is not necessary to perform this iteration, because the precise value of $a$ does not affect the quantities of interest such as the gap shape, the normal tractions in the large contact zone and the value of $T / Q$ (or $b / L$ ). It does affect a little the shear stress intensity factor at $-L$, however this stress intensity factor cannot be computed accurately in any case unless $n$ is chosen to be of the order of 100 at least, as observed also in [4] and [5]. Since the numerical computations become prohibitively expensive, no attempt was made to compute the shear stress intensity factor at $-L$.

The shear stress intensity factor at $L^{+}$defined as

$$
\mathscr{K}_{2}\left(L^{+}\right)=\lim _{x \rightarrow L}[\sqrt{ }(2(x-L)) N(x)]
$$

is related to $\phi(1)$ by

$$
\mathscr{K}_{2}\left(L^{+}\right) / Q L^{1 / 2}=\phi(1)
$$

where

$$
\phi(1)=\frac{1}{n} \sum_{i=1}^{n} \frac{\sin \left[\frac{(2 n-1)(2 i-1) \pi}{4 n}\right]}{\sin \left[\frac{(2 i-1) \pi}{4 n}\right]} \phi\left(r_{i}\right)
$$

The normal tractions are singular at $L^{-}$in the contact zone[17] and the intensity of the singularity is given by

$$
\mathscr{K}_{1}\left(L^{-}\right)=-\beta \mathscr{K}_{2}\left(L^{+}\right)
$$

Since the normal tractions must be compressive in the contact zone $\mathscr{K}_{1}\left(L^{-}\right)$must be negative, or for $\beta>0, \mathscr{K}_{2}\left(L^{+}\right)$must be positive.

We now turn to the numerical computation of the kernels $K_{1}, K_{2}, K_{3}$ given by eqns (17)-(19). These kernels are integrals with semi-infinite range and integrands with oscillatory behavior which, however, decay exponentially. When the frequency of the oscillations is small, the Laguerre quadrature is very effective, see Stroud and Secrest[15]. When the frequency is large, we must use quadrature rules especially designed for oscillatory functions, (Davis and Rabinowitz[16]). The Laguerre integration for 33 points was compared with the quadrature of Krylov and Skoblya ([16], eqns (3.9.13) and (3.9.14)) for 256 points and step size equal to 0.0625 . It was found that for frequencies of oscillation up to 4 the Laguerre integration is better, while for higher frequencies the Krylov-Skoblya integration is better in terms of accuracy. In our case the frequency of the sinusoidal terms is $(\xi-x) / h$ and has a maximum value of $2 L / h$. Therefore, the Laguerre integration is preferable for values of $L / h$ up to 2 .

The material combination $\alpha=1, \beta=0.5$ corresponds to a rigid layer bonded to an elastic half space, which is identical with the problem treated in [2]. Since the method of [2] could not be employed here for other material combinations, this case provided a check for the present method. 


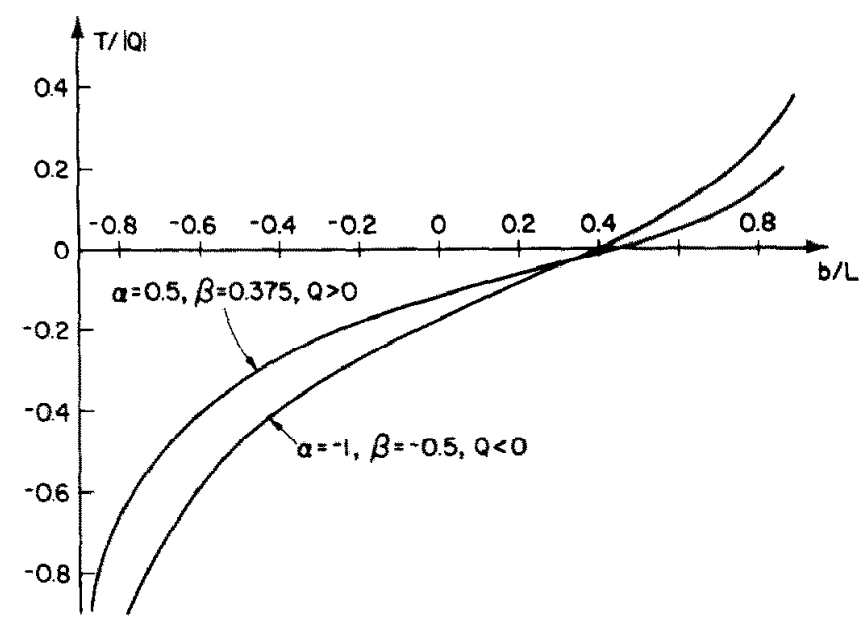

Fig. 3. Dependence of $b / L$ on $T / Q$ for $L=0.5$.

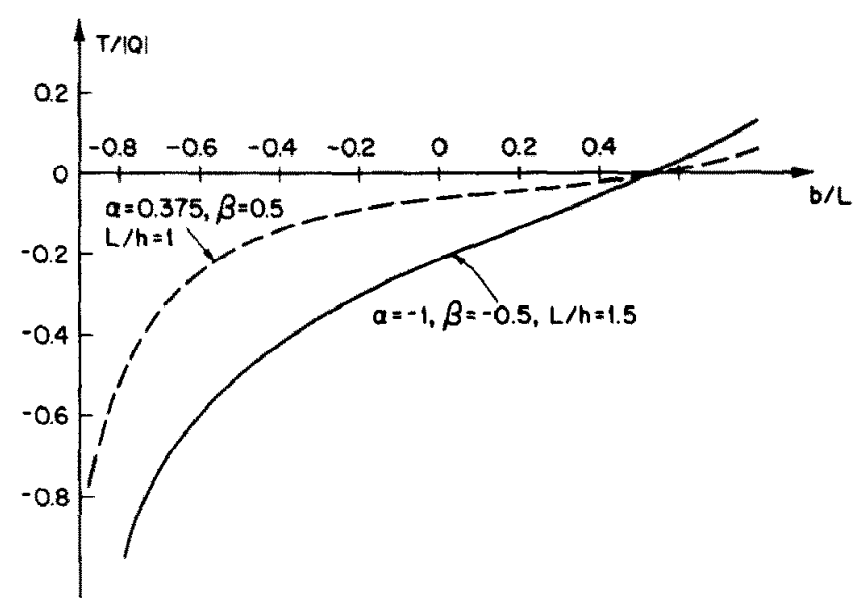

Fig. 4. Dependence of $b / \mathrm{L}$ on T/Q. Solid line: $\alpha=-1, \beta=-0.5, L / h=1.5$. Dotted line: $\alpha=0.375, \beta=0.5$, $L / h=1$.

The dependence on $T / Q$ of the parameter $b / L$ specifying the large contact zone is shown in Fig. 3 for $L / h=0.5$ and the material combinations $\alpha=0.5, \beta=0.375$ and $\alpha=-1, \beta=-0.5$ (rigid substrate). Note that in the first case $Q>0(\beta>0)$ and in the second $Q<0(\beta<0)$ for the reason stated at the end of the previous section. In Fig. 4 the solid line depicts the dependence of $b / L$ on T/Q for $\alpha=-1, \beta=-0.5$ and $L / h=1.5$, while the dotted line corresponds to $\alpha=0.375, \beta=0.5$ and $L / h=1$. For $L / h=1.5$ the latter material combination produced overlapping starting at values of $b / L$ between 0.8 and 0.85 . The overlapping indicates that a third detached contact zone must be introduced in the vicinity of overlapping and the problem must be reformulated and solved anew. This result is not unexpected since $\alpha=0.5, \beta=0.375$ and $L / h=1.5$ correspond to a flexible and relatively thin layer. It is interesting to note that, before overlapping occurs, the gaps form already a dimple even for small positive $T$. The dimple leads to additional contact as $T$ becomes compressive. Such gaps are shown in Fig. 5 corresponding to $b / L=0.9, T / Q=0.032$ and $b / L=0.85, T / Q=0.0037$. Figure 6 shows gaps for $\alpha=0.5, \beta=0.375$ and $L / h=1$ and 1.25 corresponding to $T / Q=-0.24$ and $T / Q=-0.027$ respectively (compression). In Fig. 7 the solid line shows the gap for $\alpha=0.5, \beta=0.375, T / Q=-0.394$ and the dotted line shows the gap for $\alpha=-1, \beta=-0.5, T /|Q|=-0.584$. Both gaps correspond to $L / h=0.5$ and $b / L=-0.6$. The stress intensity factor $\mathscr{K}_{2}\left(L^{+}\right)$shows little variation with $b / L$. Thus, for the material combination $\alpha=0.5$, $\beta=0.375, \mathscr{K}_{2}\left(L^{+}\right) / Q L^{1 / 2}$ increases from 1.065 to 1.077 for $L / h=0.5$ and from 1.14 to 1.17 for $L / h=1$ as 


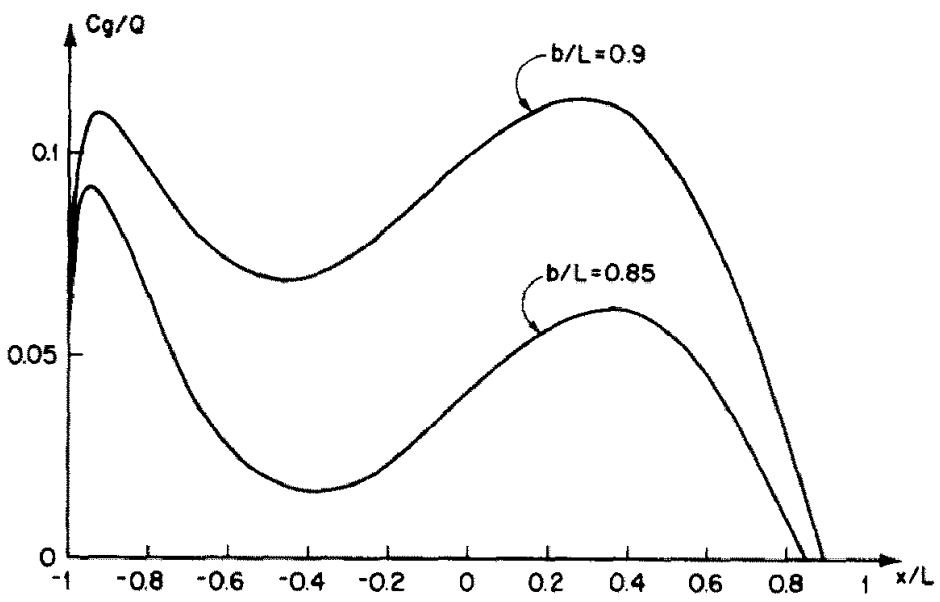

Fig. 5. Gap shapes for $\alpha=0.5, \beta=0.375, L / h=1.5$.

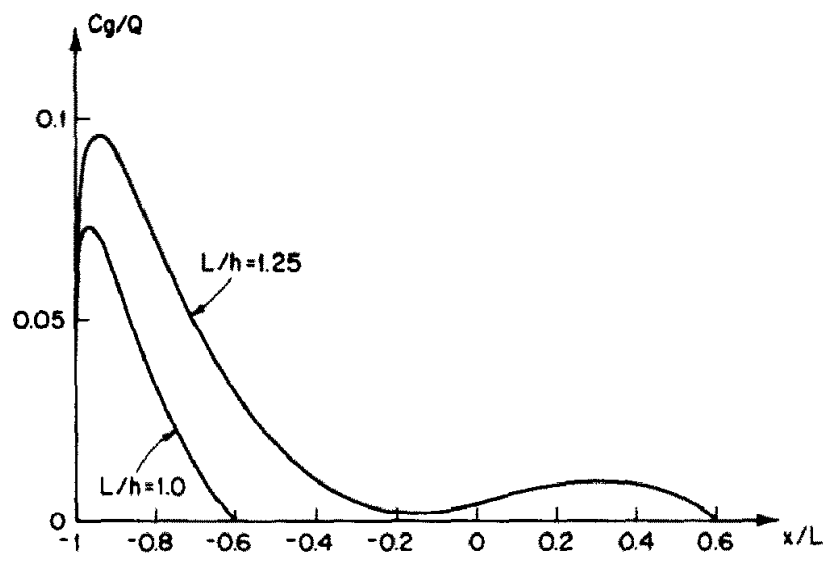

Fig. 6. Gap shapes for $\alpha=0.5, \beta=0.375, L / h=1.25$ and $L / h=1.0$

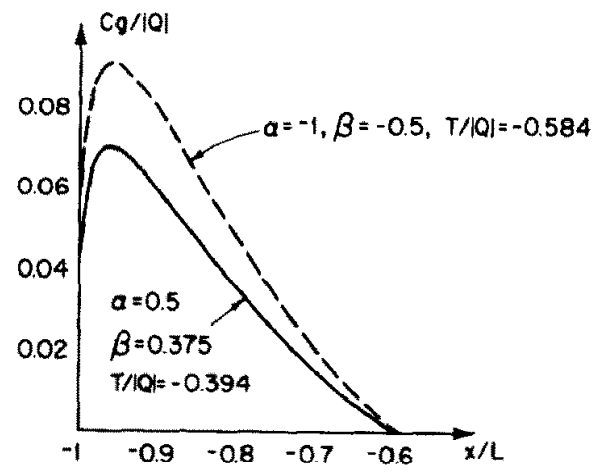

Fig. 7. Gap shapes for $b / L=-0.6, L / h=0.5$. Solid line: $\alpha=0.5, \beta=0.375$. Dotted line: $\alpha=-1, \beta=-0.5$.

$b / L$ varies from 0.8 to -0.8 . For the material combination $\alpha=-1, \beta=-0.5, \mathscr{T}_{2}\left(L^{+}\right) /|Q| L^{1 / 2}$ varies from -1.13 to -1.02 for $L / h=0.5$, and from -1.10 to -1.14 for $L / h=1.5$ as $b / L$ varies from 0.8 to -0.8 .

Although the problem has not been exhausted and a complete parametric study was not undertaken, it is hoped that its salient features have been sufficiently presented. 


\section{REITRENCES}

[1] M. Comninou, The interface crack in a shear field. J. Appl. Mech. 45, 287-290 (1978).

[2] M. Comninou and D. Schmueser, The interface crack in a combined tension-compression and shear field. J. Appl. Mech. 46, 345-348 (1979).

[3] M. Comninou and J. Dundurs, Effect of friction on the interface crack loaded in shear. J. Elasticity 10, 203-212 (1980).

[4] M. Comninou and F.-K. Chang, The interface crack in a shear and bending field. In 1981 Advances in Aerospace Structures and Materials (Edited by S. S. Wang and W. J. Renton), ASME, New York (1981).

[5] M. Comninou, Exterior interface cracks. Int. J. Engng Science 18, 501-506 (1980).

[6] W. Janach and L. H. Guex, In-plane propagation of shear microcracks in brittle rocks under triaxial compression. J. Geophysical Research 85, 2543-2553 (1980).

[7] K. M. Liechti and W. G. Knauss, The use of crack profile measurements to determine mode interactions of propagating cracks of material interfaces. In 1981 Advances in Aerospace Structures and Materials (Edited by S. S. Wang and W. J. Renton), ASME, New York (1981).

[8] J. Dundurs and M. Comninou, Some consequences of the inequality conditions in contact and crack problems. J. Elasticity 9 , $71-82$ (1979).

[9] J. K. Knowles and E. Sternberg, On the singularity induced by certain mixed boundary conditions in linearized and nonlinear elastostatics. Int. J. Solids Structures 11, 1173-1201 (1975).

[10] R. Weeks, J. Dundurs and M. Stippes, Exact analysis of an edge dislocation near a surface layer. Int. J. Engng Science 16, 365-372 (1968).

[11] M. Comninou, A property of interface dislocations. Philosophical Magazine 36, 1281-1283 (1977).

[12] J. Dundurs, Elastic interaction of dislocations with inhomogeneities. In Mathematical Theory of Dislocations (Edited by $T$. Mura). ASME, New York (1969).

[13] F. Erdogan and G. Gupta, On the numerical solution of singular integral equations. Q. Appl. Mathematics 30, 525-529 (1972).

[14] S. Krenk, On the use of the interpolation polynomial for solutions of singular integral equations. Q. Appl. Mathematics 32 , $479-484$ (1975).

[15] A. H. Stroud and D. Secrest, Gaussian Quadrature Formulas. Prentice-Hall, Englewood Cliffs, New Jersey (1966).

[16] P. J. Davis and P. Rabinowitz, Methods of Numerical Integration. Academic Press, New York (1975).

[17] M. Comninou, The interface crack. J. Appl. Mechanics 44, 631-636 (1977). 